\title{
Genetic Fourier Descriptor for the Detection of Rotational Symmetry
}

\author{
Raymond K. K. Yip \\ Department of Information and Applied Technology, Hong Kong Institute of Education \\ 10 Lo Ping Road, Tai Po, New Territories, Hong Kong
}

\begin{abstract}
In this paper, a Genetic Fourier Descriptors is proposed to detect rotational symmetry. Rotational symmetry is one of the important features for image decoding and object recognition in computer vision systems. In the Genetic Fourier algorithm, the Fourier descriptors are chromosomes and fitting function of the GA. The Genetic Fourier method has the following advantages. (1) It can handle partially occurred contour and opened contour, (2) It can handle complex point pattern and (3) It is highly parallel and its efficient can be greatly improved if parallel processors are used. Experimental results show that it can handle complex symmetry figures, these symmetry figures may be formed by separated curves, points or partially occurred or partially missed (open contour).
\end{abstract}

Key-Words: Genetic Algorithm, Fourier Descriptors, Rotational Symmetry Detection

\section{Introduction}

In this paper, a Genetic Fourier Descriptors is proposed to detect rotational symmetry. Rotational symmetry is one of the important features for image decoding and object recognition in computer vision systems

Rotational symmetry is one of the important features for image decoding and object recognition in computer vision systems. It plays an important role in pattern recognition, especially when extracting a planar symmetric figure from a single image without the need of models. In the recent years, many methods have been proposed for the detection of rotational symmetry. However most of the methods are difficult to handle complex symmetry figure, e.g. figure 5a. Some methods, like Hough transform [8] can handle these situation but they only extract the symmetric points and the problem of finding the connected contour (connectivity problem) and equation (parameterization problem) of the figure still left open.

Fourier descriptors, FDs [1-4] are one of the powerful tools for object recognition and symmetry detection. However, it cannot handle partially occurred symmetry figure, symmetric point pattern and open contour situation.

On the other hand, Genetic Algorithms (GAs) are powerful tools in the areas of computer vision. It employs the evolution process of natural selection to find (search) the optimal solution of a desired problem. GA works with a population of chromosomes, each represents a potential solution and fitness value to a desired problem. Chromosomes with the higher fitness have a better chance to progress their information to the next generation through the chromosomes crossover and gene mutation process. If a GA is designed well, the population will finally converge to an optimal solution. In this paper, Fourier descriptors are selected as the fitting function and its coefficients become the chromosomes of the GA. Experimental results show that it can handle complex symmetry figures, these symmetry figures may be formed by separated curves, points or partially occurred or partially missed (open contour).

\section{Elliptic Fourier descriptors}

Fourier descriptors (FD's) are useful in describing and recognizing the shapes of 2-D closed contours. The basic idea is, a closed curve may be represented by a periodic function of a continuous parameter $t$, or alternatively, by a set of Fourier coefficients of this function. In general, there exists infinitude ways to parameterize a 2-D closed curve to form the FD's. The reason for the infinitude is that $t$ is an 


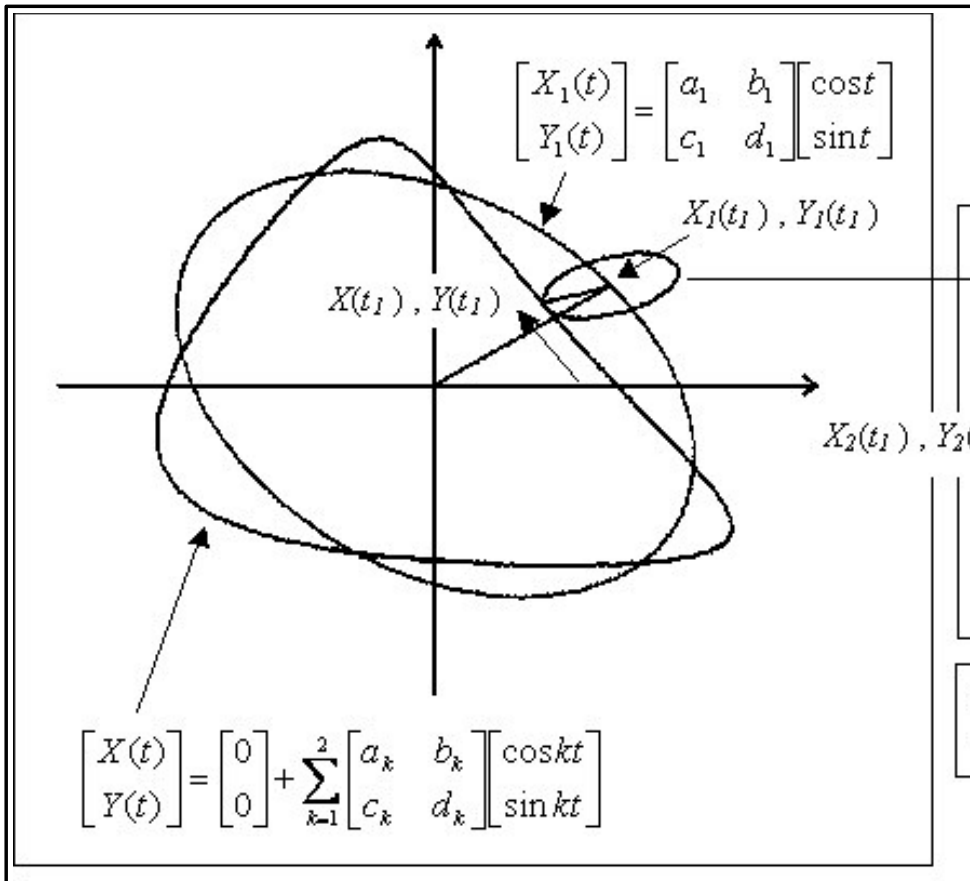

Figure 1a Graphical meaning of Fourier Descriptors with $\operatorname{maximum} k=2$.

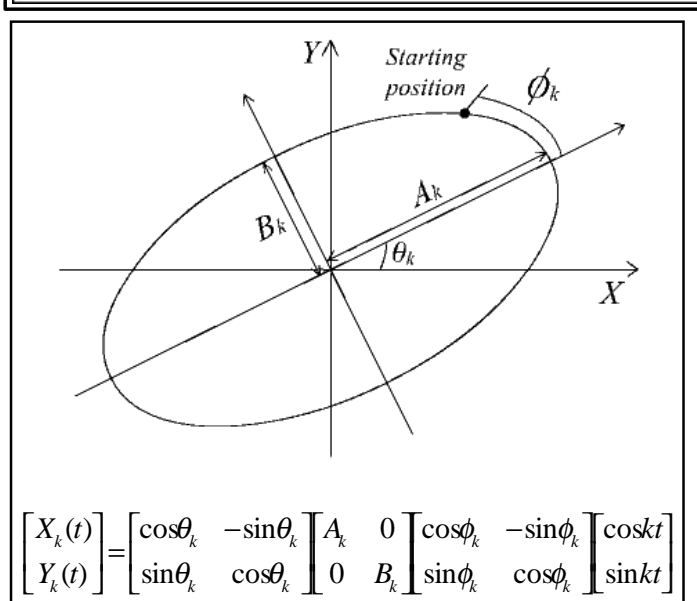

(2)

Figure 1. The rotation an starting phase of an ellipse of the $k$ th harmonic.

implicit (hidden) parameter. Therefore, there exists an infinite number of ways to distribute its values along the curve. The most common (familiar) way is based on the arc length $l(t=$ $2 \pi l / L$ where $L$ is the perimeter of the contour) parameterization. As the calculation of the parameter $t$ using arc length (constant speed assumption) is simple, this parameterization method is widely used by many researchers.

The basic idea of elliptic Fourier descriptors is as follows. A closed curve may be represented by a periodic function of a continuous parameter $t$, eqt. (1)

$$
\left[\begin{array}{l}
X(t) \\
Y(t)
\end{array}\right]=\left[\begin{array}{l}
a_{0} \\
c_{0}
\end{array}\right]+\sum_{k=1}^{\infty}\left[\begin{array}{ll}
a_{k} & b_{k} \\
c_{k} & d_{k}
\end{array}\right]\left[\begin{array}{l}
\cos k t \\
\sin k t
\end{array}\right]
$$

where $t=0$ to $2 \pi$ and $k=0,1,2, \ldots,(X(t), Y(t))$ is the coordinates of the contour in the image planes and $\left[\begin{array}{llll}a_{k} & b_{k} & c_{k} & d_{k}\end{array}\right]$ are the $k$ th coefficients of the FD.

In using Fourier descriptors for pattern recognition, a curve representation must be normalized with respect to a desired transformation domain so that the FD' $s$ are invariant with respect to the specified domain. Invariant of FDs for object recognition and symmetry detection under 2D and 3D (affine) transform have been widely investigated. However, using arc length parameterization FD for object recognition and symmetry detection has the following limitation.

(1) Image points must be connected (e.g. chain coded) to form a closed contour before FDs can be applied, this leads to great difficulties when the point patterns are complex (e.g. figure 5a)

(2) It cannot handle partially occurred object

(3) It cannot handle opened contour (e.g. figure 8b)

This paper proposes a new strategy using a Genetic-Fourier (GFD) algorithm for the extraction of rotational symmetry. The proposed method has the following advantages. 
(1) It eliminates the arc length parameterization method so it can handle partially occurred contour and opened contour

(2) It eliminates the image points' connection process so it can handle complex point pattern.

(3) It is highly parallel and its efficient can be greatly improved if parallel processors are used.

\subsection{FD Invariants of Rotational Symmetry}

Elliptic Fourier descriptors invariants of symmetry have been explored in [4]. Recall properties 1 and 2, for a $N$ order rotational symmetry, the following properties hold.

Property 1:

For the coefficients of the $k$ th harmonic,

if $k \neq n \mathrm{~N}_{\mathrm{s}}+1$ and $k \neq n \mathrm{~N}_{\mathrm{s}}-1$,

then $a_{k}=b_{k}=c_{k}=d_{k}=0$.

where $n=1,2,3, \ldots$

Property 2:

For the coefficients of the $k$ th harmonic,

if $k=n \mathrm{~N}_{\mathrm{s}}-1$, then $A_{k}=-B_{k}$

if $k=n \mathrm{~N}_{\mathrm{s}}+1$, then $A_{k}=B_{k}$

i.e. $\left|a_{k}\right|=\left|d_{k}\right|,\left|b_{k}\right|=\left|c_{k}\right|$ and $\theta_{k}=0$

where $n=1,2,3, \ldots$

From equation (1), (2), and property 1 , it can be obtained that

$\left[\begin{array}{l}X(t) \\ Y(t)\end{array}\right]=\left[\begin{array}{l}x_{0} \\ y_{0}\end{array}\right]+\sum_{k=1}^{\infty}\left[\begin{array}{cc}A_{k} & 0 \\ 0 & B_{k}\end{array}\right]\left[\begin{array}{c}\cos \left(k t+\phi_{k}\right) \\ \sin \left(k t+\phi_{k}\right)\end{array}\right]$

where $x_{0}=a_{0}, y_{0}=c_{0}$,

From properties 2, it can be obtained that

$$
\begin{gathered}
{\left[\begin{array}{l}
X(t) \\
Y(t)
\end{array}\right]=\left[\begin{array}{l}
x_{0} \\
y_{0}
\end{array}\right]+\sum_{n=1}^{\infty}\left\{A_{n N-1}\left[\begin{array}{cc}
1 & 0 \\
0 & -1
\end{array}\right]\left[\begin{array}{l}
\cos \left((n N-1) t+\phi_{n N-1}\right) \\
\sin \left((n N-1) t+\phi_{n N-1}\right)
\end{array}\right]\right.} \\
\left.+A_{n N+1}\left[\begin{array}{ll}
1 & 0 \\
0 & 1
\end{array}\right]\left[\begin{array}{l}
\cos \left((n N+1) t+\phi_{n N+1}\right) \\
\sin \left((n N+1) t+\phi_{n N+1}\right)
\end{array}\right]\right\}
\end{gathered}
$$

Hence, the detection of a $N$ order rotational symmetry can be considered as finding the best fit for the FD parameters $\left(x_{0}, y_{0}, A_{l}, \phi_{l}, A_{N-l}\right.$, $\phi_{N-1}, A_{N+1}, \phi_{N+1}, \ldots, A_{n N-1}, \phi_{n N-1}, \quad, A_{n N+1}$, $\left.\phi_{n N+1}, \ldots\right)$.

\section{Genetic Algorithm (GA)}

GA is a searching (optimization) method using procedures that are inspired by natural evolution, it involves population (chromosomes), mating, crossover, mutation, natural selection (fitness measure) and generation (iteration).

The population consists of fixed- or variable-length chromosomes (potential solutions). The chromosomes are formed by real value or binary format to represent the parameters of the problem domain. Figure 2 shows that there are $p$ chromosomes in the population. In each update,

1) $b$ chromosomes are selected and remain in the population, usually they are the first $b$ best fit (FM) chromosomes.

2) $m$ chromosomes are selected for mutation and produces $m$ new chromosomes (self reproduction),

3) $c_{p}$ pairs chromosomes are selected for crossover to produces $n$ new chromosomes, these $n$ new chromosomes with further go to the mutation process. In general, $n$ is either equal to $c_{p}$ (2 to 1 ) or $2 c_{p}$ (2 to 2 ).

4) All the new chromosomes produced in the mutation process will then perform the fitness measure and $q$ new chromosomes will be used to update the population. In general $q=m+n$.

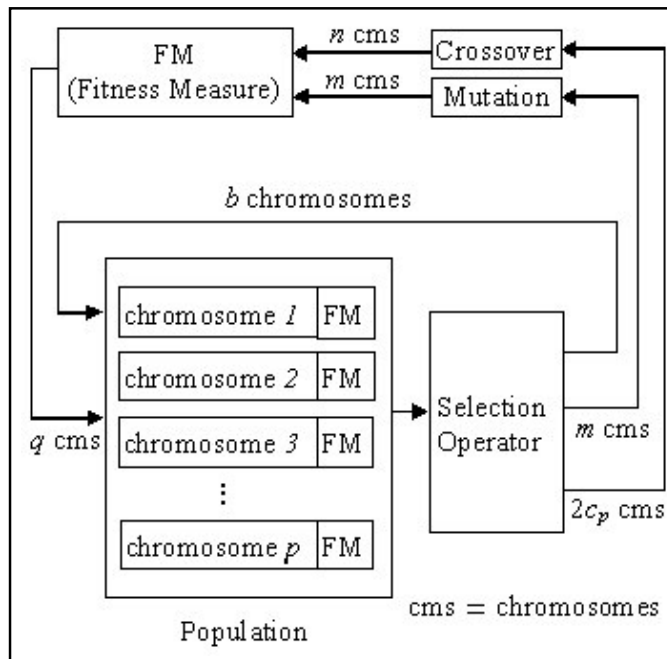

Figure 2. The general flow of genetic algorithm.

In general, the number of population, $p$ can be fixed or varies. If $p$ is fixed, then $q=p-b, n$ $=p-b-m$.

The selection mechanism takes many forms, yet it always ensures that the best fit chromosomes have a higher probability to be selected for reproduction. 


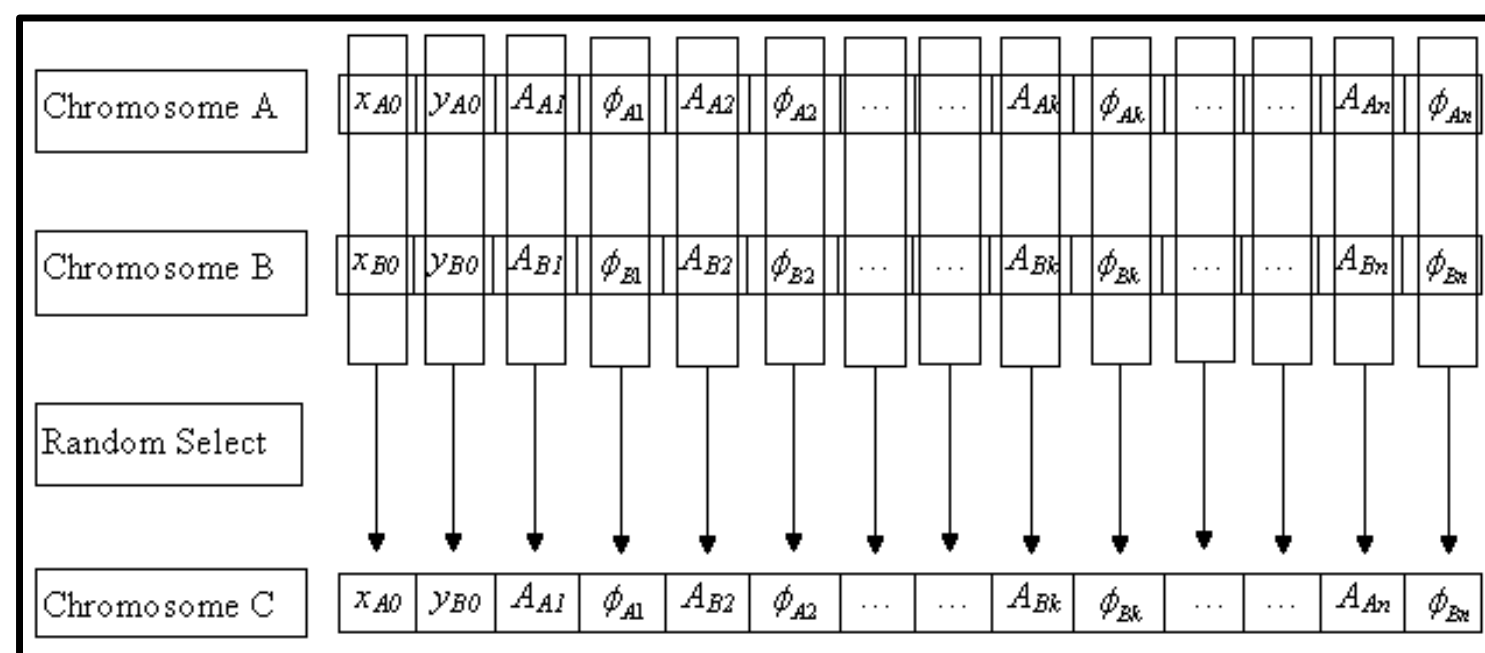

Figure 3. Figure indicates the crossover process.

\subsection{Defining genetic chromosomes, parameters and operators}

Coding chromosomes: The chromosome of the GFD is formed by the parameters of the FDs, i.e. $\left(x_{0}, y_{0}, A_{1}, \phi_{1}, A_{2}, \phi_{2}, \ldots, A_{k}, \phi_{k}, \ldots, A_{20}\right.$, $\left.\phi_{20}\right)$ with the maximum harmonic being 20 . Here, real value is used.

Selection: selection operator is used to select good chromosomes for the progress of their inherited knowledge to the next generation. In the proposed algorithm, the selection probability is proportional to the square root of its fitness value.

Direct selection: It is used to maintain those chromosomes that have the highest chance to success. In this paper, the top $l$ chromosomes that have the best fit will be maintained in the population.

Crossover: Crossover operator is used to produce new chromosomes through combining partial structure of two father individuals. Figure below shows the crossover mechanism of the proposed algorithm.

Mutation: In order to prevent the loss of diversity in the evolutionary process, a mutation process is designed and operates to represent a sudden change in a small area of a chromosome. The mutation process will directly obtain a small among $(m)$ of chromosomes from the population. In this paper, the top $m$ chromosomes that have the best fit will be selected. The probability $P_{m}$ of the individual FD coefficients undergo the mutation process is set to 0.5 with the following process is used for the selected FD coefficients

$$
\begin{aligned}
& A_{k}=A_{k}+32(\text { Rnd }-0.5) \\
& \phi_{k}=\phi_{k}+32(\text { Rnd }-0.5)
\end{aligned}
$$

where Rnd is a random generator between 0 to 0.999 .

Fitness function: In this paper, the following fitness function is used.

$$
F D_{\text {fitness }}=F D_{\text {energy }} \frac{F D_{\text {energy }}}{5 F D_{\text {pts }}}
$$

$F D_{\text {energy }}$ is an energy function represents a distances measure between the FD and the image points. $F D_{p t s}$ is the number of image pixels passed by the FD and $F D_{\text {energy }} / 5 F D_{\text {pts }}$ is a completeness measure.

Consider figure 4 , figure 4 a shows a $7 \times 7$ image with (1) 7 image points and (2) a FD curve passing through it. Figure $4 \mathrm{~b}$ shows the energy measure of the figure $4 a$, the position of an image point will have the highest value 5 and the value will gradually decrease as the distance is away from the image points. For figure 4 ,

\begin{tabular}{|c|c|c|c|c|c|c|}
\hline 1 & & & 2 & & & \\
\hline & 1 & & 2 & & & \\
\hline & & 1 & 2 & & & \\
\hline & & & 12 & & & \\
\hline & & & 2 & 1 & & \\
\hline & & & 2 & & 1 & \\
\hline & & & 2 & & & 1 \\
\hline
\end{tabular}

Fig.4a. (1) Image points and (2) the FD curve .passing through the image. 


\begin{tabular}{|c|c|c|c|c|c|c|}
\hline 5 & 2.8 & 1.5 & 0 & 0 & 0 & 0 \\
\hline 2.8 & 5 & 2.8 & 1.5 & 0 & 0 & 0 \\
\hline 1.5 & 2.8 & 5 & 2.8 & 1.5 & 0 & 0 \\
\hline 0 & 1.5 & 2.8 & 5 & 2.8 & 1.5 & 0 \\
\hline 0 & 0 & 1.5 & 2.8 & 5 & 2.8 & 1.5 \\
\hline 0 & 0 & 0 & 1.5 & 2.8 & 5 & 2.8 \\
\hline 0 & 0 & 0 & 0 & 1.5 & 2.8 & 5 \\
\hline
\end{tabular}

Fig. 4b. Energy measures of figure $4 \mathrm{a}$.

$F D_{\text {energy }}=1.5+2.8+5+2.8+1.5=13.6$,

$F D_{p t s}=7$.

Therefore, $F D_{\text {fitness }}=13.6 \times 13.6 /(5 \times 7)=5.28$. The factor $F D_{\text {energy }} / 5 F D_{\text {pts }}$ is a completeness measure of the corresponding FD.

Parameter and Initial setting: In this paper, the following parameters are used. $P=100, b=10$, $m=10, c_{p}=80$ and $n=80 . N$ is fixed at the beginning of the iteration and the initial values of $x_{0}, y_{0}, A_{k}$ and $\phi_{k}$ are set using the following processes.

$A_{1}$ is randomly selected between 0 to 128 (for a 256 x 256 image)

$\phi_{I}$ is randomly selected between 0 to $360^{\circ}$

$\theta$ is randomly selected between 0 to $360^{\circ}$

$x_{0}=I_{i}(x)+A_{l} \cos \phi_{1} \quad y_{0}=I_{i}(y)+A_{l} \sin \phi_{l}$

$A_{k}=0 \quad \forall k>0 \quad \phi_{k}=0 \quad \forall k>0$

where $I_{i}$ is an image point randomly selected from the input image and $I_{i}(x)$ and $I_{i}(y)$ are the corresponding $\mathrm{x}$ and $\mathrm{y}$ co-ordinators of the $i$ image.

\section{Experimental Results}

In order to verify the proposed algorithm, a lot of experiments have been performed. This section presents some of these experiments.

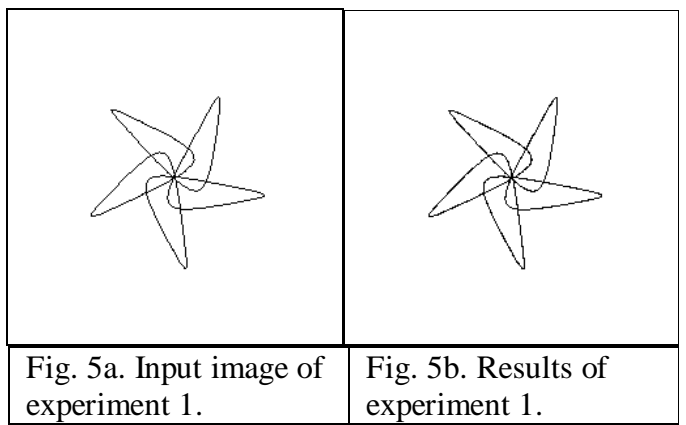

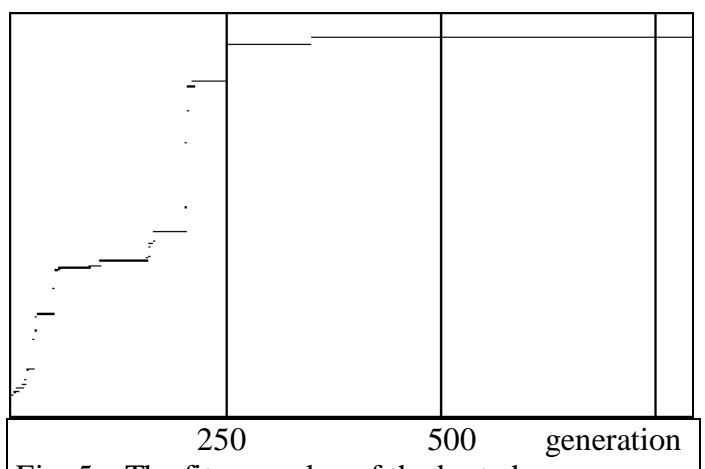

Fig. 5c. The fitness value of the best chromosome.

\begin{tabular}{|c|c|c|c|c|c|c|c|c|}
\hline$N$ & $x_{0}$ & $y_{0}$ & $A_{1}$ & $\phi_{1}$ & $A_{4}$ & $\phi_{4}$ & $A_{6}$ & $\phi_{6}$ \\
\hline 5 & 128 & 128 & 30 & $0^{\circ}$ & 30 & $16^{\circ}$ & -12 & $-36^{\circ}$ \\
\hline
\end{tabular}

Table 1. FD coefficients obtained in experiment 1.
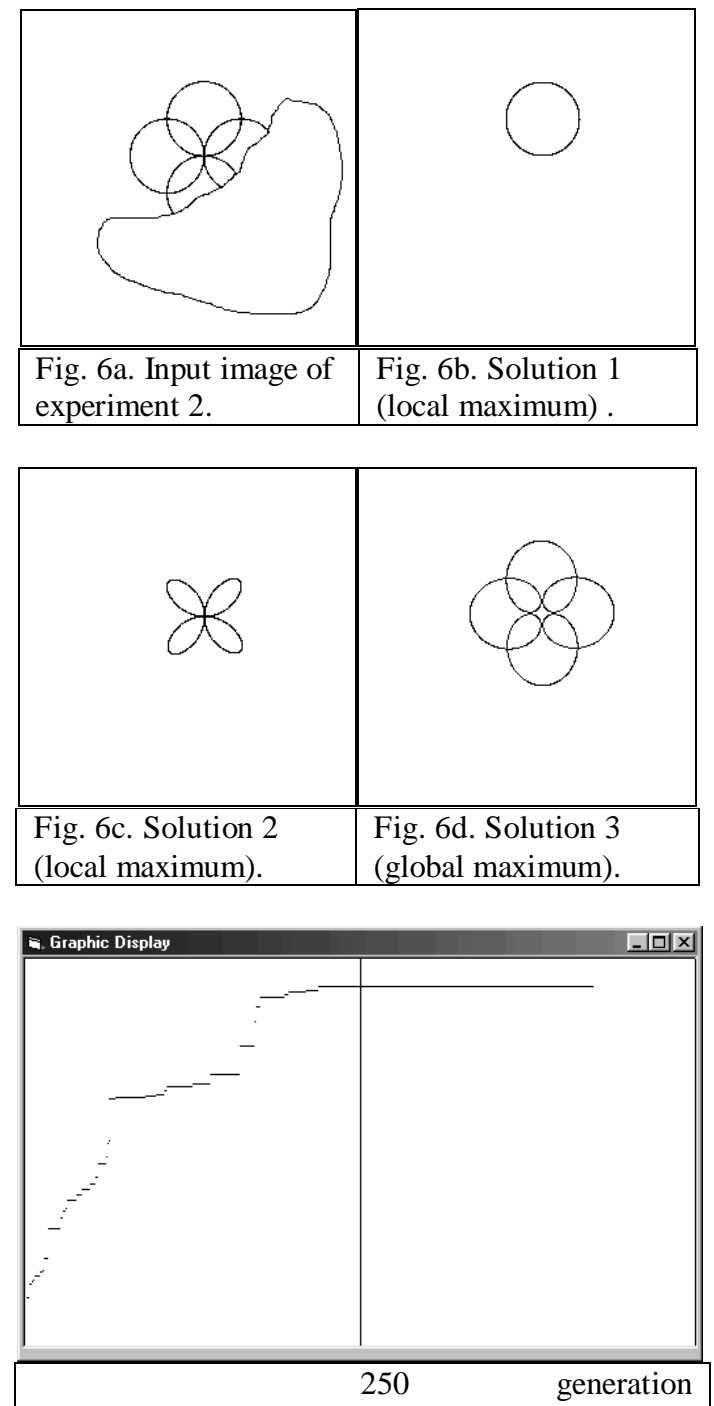

Fig. 6e. The fitness value of the best chromosome of $6 \mathrm{~d}$. 


\begin{tabular}{|c|c|c|c|c|c|c|c|c|c|c|c|c|c|}
\hline Solution & $N$ & $x_{0}$ & $y_{0}$ & $A_{1}$ & $\phi_{1}$ & $A_{4}$ & $\phi_{4}$ & $A_{6}$ & $\phi_{6}$ & $A_{4}$ & $\phi_{4}$ & $A_{6}$ & $\phi_{6}$ \\
\hline 1 & 5 & 140 & 83 & 28 & $0^{\circ}$ & 0 & $0^{\circ}$ & 0 & $0^{\circ}$ & 0 & $0^{\circ}$ & 0 & $0^{\circ}$ \\
\hline 2 & 5 & 140 & 111 & 20 & $0^{\circ}$ & 8 & $180^{\circ}$ & 9 & $0^{\circ}$ & 0 & $0^{\circ}$ & 0 & $0^{\circ}$ \\
\hline 3 & 5 & 140 & 111 & 31 & $0^{\circ}$ & 5 & $0^{\circ}$ & 20 & $180^{\circ}$ & 1 & $180^{\circ}$ & 1 & $0^{\circ}$ \\
\hline
\end{tabular}

Table 3. FD coefficients obtained in experiment 2.

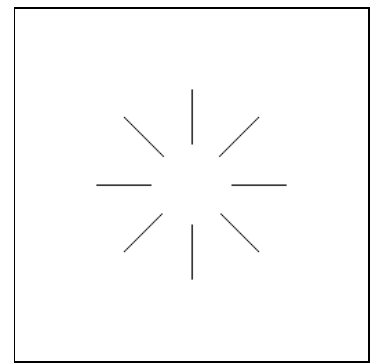

Figure 7a. Input image of experiment 3

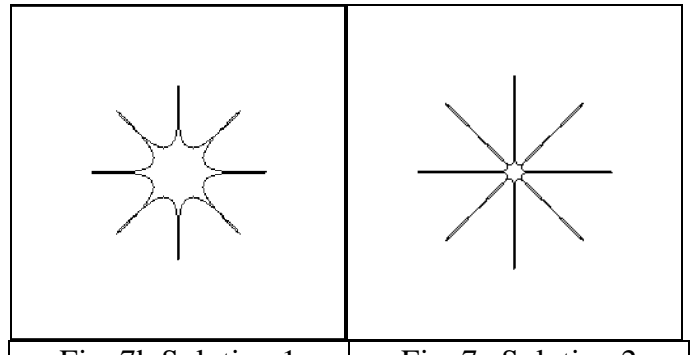

Fig. 7b Solution 1

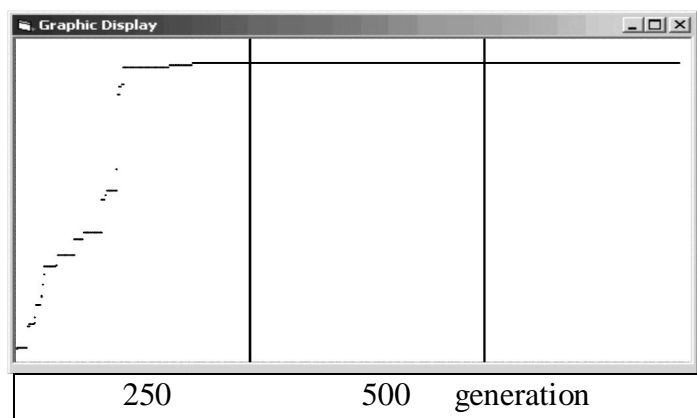

Fig. 7d. The fitness value of the best chromosome of $7 \mathrm{c}$.

\begin{tabular}{|c|c|c|c|c|c|c|c|c|c|}
\hline$N$ & $\theta$ & $x_{0}$ & $y_{0}$ & $A_{1}$ & $\phi_{1}$ & $A_{7}$ & $\phi_{7}$ & $A_{9}$ & $\phi_{9}$ \\
\hline 8 & $0^{\circ}$ & 128 & 128 & 44 & $0^{\circ}$ & 16 & $0^{\circ}$ & -7 & $0^{\circ}$ \\
\hline
\end{tabular}

Table 3. FD coefficients of figure $7 \mathrm{~b}$.

\begin{tabular}{c|c|c|c|c|c|c|c|c|c|c|}
\hline$N$ & $\theta$ & $x_{0}$ & $y_{0}$ & $A_{1}$ & $\phi_{1}$ & $A_{7}$ & $\phi_{7}$ & $A_{9}$ & $\phi_{9}$ \\
\hline 8 & $0^{\circ}$ & 128 & 128 & 40 & $0^{\circ}$ & 21 & $0^{\circ}$ & -13 & $0^{\circ}$ \\
\hline
\end{tabular}

Experiment 1 and 2 show that the algorithm performed well for complex symmetry figure. Experiment 3 shows that the algorithm can handle symmetry formed by separate curves and different solutions (interpretation) may be obtained. Experiment 4 shows that the GFD can handle symmetry figure that has missing portion.

\section{Conclusion}

In this paper, a Genetic-Fourier algorithm is proposed for the detection of rotational symmetry. The proposed method has the following advantages.

(1) It can handle partially occurred contour and opened contour

(2) It can handle complex point pattern.

(3) It is highly parallel and its efficient can be greatly improved if parallel processors are used.

\section{References}

[1] C.T. Zhan and R.Z. Roskies, "Fourier descriptors for plane closed curves", IEEE Trans. Comput., vol. C-21, no. 3, pp.269281, Mar. 1972.

[2] G H Granlund, "Fourier preprocessing for hand print character recognition", IEEE Trans. Comput., vol. C-21, pp.195-201, Feb. 1972.

[3] C.S. Lin and C. L. Hwang, "New forms of shape invariants from elliptic Fourier descriptors", Pattern Recognition, Vol. 20, no. 5, pp. 535-545, 1987.

[4] KK Yip and K S Tam, "Application of Elliptic Fourier Descriptors to Symmetry Detection Under Parallel Projection", IEEE Trans. on PAMI, vol. 16, No. 3, March 1994.

[5] L Zhang, W Xu and C Chang, "Genetic algorithm for affine point pattern matching", Pattern Recognition Letters 24, pp. 9 -19, 2003.

[6] K P Han, K W Song, E Y Chung, S J Cho, Y $\mathrm{H} \mathrm{Ha}$, "Stereo matching using genetic algorithm with adaptive chromosomes", Pattern Recognition 34, pp. 1729 -1740, 2001

[7] S Y Yuen and C H Ma, "Genetic algorithm with competitive image labelling and least square", Pattern Recognition 33, pp. 1949 1966, 1999.

[8] R.K.K. Yip, "A Hough Transform Technique for the Detection of Parallel Projected Rotational Symmetry", Pattern Recognition Letters, Vol. 20, pp.991 - 1004, 1999. 